\title{
Sequential adjustments before and after partial errors
}

\author{
Sonia Allain \\ Institut de Médecine Navale du Service de Santé des Armées, Toulon, France \\ Boris BURle AND ThIERRY HasbroucQ \\ CNRS and Université de Provence, Marseille, France \\ AND \\ Franck VidaL \\ CNRS and Université de Provence, Marseille, France \\ and Institut de Médecine Navale du Service de Santé des Armées, Toulon, France
}

\begin{abstract}
In choice reaction time tasks, subjects speed up before making an error, but slow down afterward to prevent the occurrence of a new error. In some trials, the correct response is preceded by an incorrect electromyographic (EMG) activation too small to reach the response threshold. In this article, we show that these incorrect EMG activations give rise to the same sequential effects as overt errors: Before a trial containing an incorrect EMG activation, subjects speed up, whereas after that trial, they slow down. These activations reflect errors that have been detected, inhibited, and corrected in time. As such, they index the involvement of online executive control.
\end{abstract}

It has been proposed that performance is controlled by a supervisory system (Botvinick, Braver, Barch, Carter, \& Cohen, 2001; Rabbitt, 1966) that monitors information processing. This supervisory system can trigger strategic adjustments of behavior to optimize task performance. In reaction time (RT) tasks, Rabbitt reported "post-error slowing"; after making an error, subjects become more cautious, in order to prevent new errors. On average, the RTs of correct trials following an error increase while error rates decrease (Laming, 1979b). Conversely, subjects are less cautious on trials preceding an error: The mean RTs of such trials are shorter than the mean RTs of correct responses preceding a correct response (Smith \& Brewer, 1995). Henceforth, this effect will be called preerror speeding. Post-error slowing and pre-error speeding can be considered sequential effects.

On certain correct trials in between-hand choice RT tasks, the electromyographic (EMG) activity of the agonist involved in the required response is preceded by EMG activity of the agonist involved in the alternative, incorrect response; there is general agreement that these trials reflect small incorrect EMG activations at a level too small to reach the response threshold (Coles, Gratton, Bashore, Eriksen, \& Donchin, 1985; Eriksen, Coles, Morris, \& O’Hara, 1985; Smid, Mulder, \& Mulder, 1990). As such, we will henceforth call these activations partial errors. Correct trials containing a partial error cannot be considered purely correct, so they will here be termed partial- error trials. Correct trials not preceded by a partial error will be named pure-correct trials.

Since it is well established that post-error slowing follows and pre-error speeding precedes full errors, one can wonder whether partial errors, which do not result in an overt failure, produce comparable sequential effects. In other words, is the executive control system able to function in a graded manner, so that it can trigger sequential adjustments proportional to the size of the dysfunction encountered in information-processing operations, or does it function instead in an all-or-none manner? This question makes sense in the context of contemporary models of executive control, in particular the conflict loop theory (Botvinick et al., 2001), whose core idea is that a conflictmonitoring module measures online the degree of conflict. Conflict is defined as the sum of the products of the activations of the possible responses weighted by the negative connection strengths between them. If only one response is activated, there is no conflict, but as soon as at least two responses are activated, conflict occurs. According to this model, when an error is committed, both responses are activated during a brief period following the incorrect response, leading to a large amount of conflict. Therefore, according to Botvinick et al., "periods of high conflict lead to a reduction in response priming resulting in slower but more accurate responding," explaining the post-error slowing. In a partial-error trial, the two responses are also activated in the same trial, so conflict does occur, but less

S. Allain, allainsonia@hotmail.com 
so than in a full-blown error trial. Nevertheless, the period of moderate conflict should lead to adjustment of the RT on the next trial, but less so than after error.

To examine these questions, in a between-hand choice RT task we compared the RTs of pure-correct trials that occurred before and after partial-error trials with the RTs of pure-correct trials that occurred before and after other pure-correct trials. To determine the role of executive control in these RT results, we manipulated both the emphasis put on accuracy and the compatibility of stimulusresponse mapping through the task instructions. Executive control is known to depend on these two factors, so our manipulations should reveal whether it is responsible for any strategic adjustments we see across trials.

\section{METHOD}

\section{Subjects}

Twelve subjects ( 4 women, 8 men; age range $23-52$ years) volunteered for the experiment. All were right-handed with normal or corrected-to-normal vision.

\section{Design and Stimuli}

The subjects were required to respond to a response signal as soon and as accurately as possible by pressing the left or the right button on a response pad (Neuroscan; Compumedics Ltd.) with their left or right thumb. When subjects produced a response, they had as feedback both the somatosensory information from the thumb movement of the buttonpress and auditory information in the form of a little "click" generated when the button was pressed. The response signals were the French words droite ("right") and gauche ("left") presented in the center of a computer screen (total visual angle, $2.14^{\circ}$ ). The stimulus-response mapping was either compatible - a response with the right thumb to the signal droite and with the left to gauche-or incompatible - responding with the right thumb to gauche and with the left to droite. In half of the blocks, subjects received an instruction emphasizing accuracy, whereas in the other half, they received an instruction emphasizing speed. The order of the four conditions (accurate-compatible, accurate-incompatible, speeded-compatible, or speeded-incompatible) was balanced across subjects. Finally, subjects were told that correcting their errors served no purpose. ${ }^{1}$

Blocks comprised 128 trials each. The subjects performed 1 training block for each compatibility mapping and then 12 experimental blocks ( 3 in each condition). The response signal was extinguished by the response, and $500 \mathrm{msec}$ later the next response signal was delivered. RTs shorter than $130 \mathrm{msec}$ or longer than $780 \mathrm{msec}$ were discarded. When subjects failed to respond within $780 \mathrm{msec}$, the response signal was extinguished and the next response signal was delivered $500 \mathrm{msec}$ later. Subjects were informed that responses occurring too late were not taken into account.

\section{Electrophysiological Recordings}

An electromyogram was recorded from the flexor pollicis brevis of each hand via paired surface $\mathrm{Ag} / \mathrm{AgCl}$ electrodes, amplified (5,000 times), filtered (bandwidth, $10 \mathrm{~Hz}-1 \mathrm{kHz}$ ), full-wave rectified, integrated (5-msec window), and then digitized (sampling rate, $256 \mathrm{~Hz}$ ). Although automated algorithms have proved to be useful, we detected EMG onsets by visual inspection of each trial because human pattern recognition processes are superior to automated algorithms (Hasbroucq, Possamaï, Bonnet, \& Vidal, 1999; Staude, 2001; Van Boxtel, Geraats, Van den Berg-Lenssen, \& Brunia, 1993). The trace corresponding to the EMG activity was displayed on the computer screen, and the EMG activity onset was marked by an experimenter unaware of the nature (correct or incorrect) of the trial that was being looking at.

Electroencephalographic activity was also recorded during this experiment for another study.

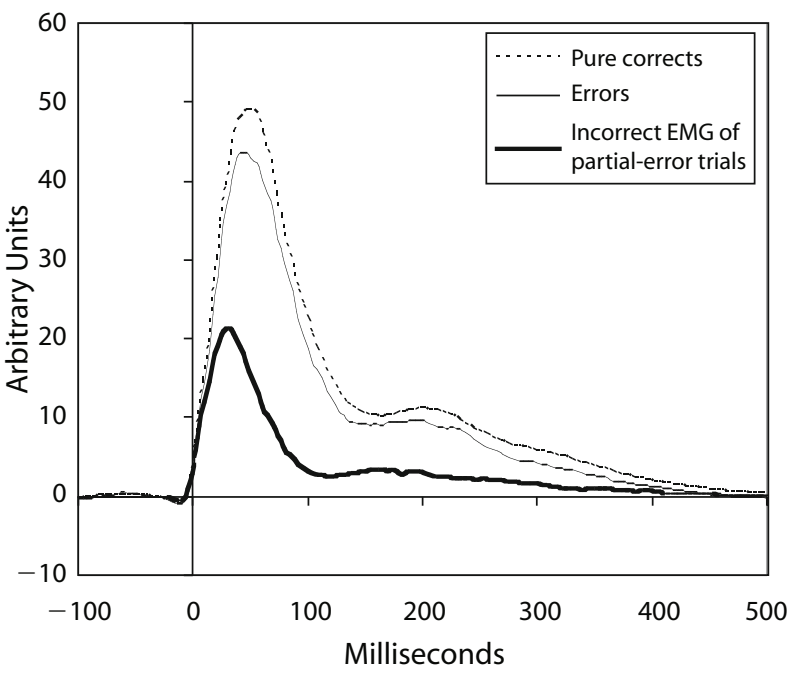

Figure 1. Electromyographic (EMG) activity averages (in arbitrary units) for pure-correct trials (dashed line), erroneous trials (thin line), and EMG latencies of incorrect responses on partialerror trials (thick line) as a function of time (abscissa, in milliseconds). Time zero corresponds to EMG onset.

\section{Classification of Trials}

Correct trials were categorized according to whether or not the activation of the agonist involved in the required response was preceded by an activation of the agonist involved in the alternative response. In this way, trials were sorted into three categories: pure correct, partial error, and error (Figure 1).

\section{Data Analysis}

The chronometric indices analyzed in the present study were the RT (the time separating the stimulus presentation from the mechanical response) and the latency of the partial error (the time separating the stimulus presentation from the onset time of the incorrect EMG activity on partial-error trials). Each measure was submitted to an ANOVA. The within-subjects variables were nature of the current trial (pure correct, partial error, or error); compatibility (compatible or incompatible); speed-accuracy instruction; and, for more specific analysis of the pure-correct RTs, accuracy (pure correct, partial error, or error) of both the preceding and the subsequent trial.

\section{RESULTS}

\section{Reaction Times}

See Table 1, top rows. RTs differed according to the nature of the response $[F(2,22)=461, p<.001]$ : Errors were faster than pure-correct responses $[F(1,11)=107, p<$ $.001]$, and pure-correct responses were faster than partial errors $[F(1,11)=407, p<.001]$. RTs were longer on incompatible than on compatible trials $[F(1,11)=10.9, p<.01]$, and the interaction between the nature of the current trial and compatibility was marginally significant $[F(2,22)=$ $3.2, p=.06]$, suggesting that the compatibility effect was less pronounced for pure-correct trials. RTs were shorter in speed-emphasized than in accuracy-emphasized conditions $[F(1,11)=16.5, p<.01]$, and the interaction between the nature of the current trial and the speed-accuracy instruction was significant $[F(2,22)=17.1, p<.001]$, indicating that the speed-accuracy instruction had a more pronounced effect on errors than on correct responses. 
Table 1

Reaction Times (RTs, in Milliseconds) and Standard Errors for Pure-Correct Trials, Erroneous Trials, and Partial-Error Trials (With RT of the Correct Hand and Latency of Electromyogram [EMG] Onset of the Incorrect Hand), As Well As Percentages of Erroneous and Partial-Error Trials, in the Four Experimental Conditions

\begin{tabular}{|c|c|c|c|c|c|c|c|c|c|}
\hline & \multicolumn{2}{|c|}{$\begin{array}{l}\text { Accurate- } \\
\text { Compatible }\end{array}$} & \multicolumn{2}{|c|}{$\begin{array}{l}\text { Speeded-- } \\
\text { Compatible }\end{array}$} & \multicolumn{2}{|c|}{$\begin{array}{c}\text { Accurate- } \\
\text { Incompatible }\end{array}$} & \multicolumn{2}{|c|}{$\begin{array}{c}\text { Speeded- } \\
\text { Incompatible }\end{array}$} & \multirow[b]{2}{*}{ Mean } \\
\hline & $M$ & $S E$ & $M$ & $S E$ & $M$ & $S E$ & $M$ & $S E$ & \\
\hline Pure-correct trial RT & 391 & 35 & 370 & 36 & 427 & 53 & 397 & 45 & 396 \\
\hline Erroneous trial RT & 337 & 36 & 299 & 31 & 393 & 72 & 341 & 57 & 343 \\
\hline \multicolumn{10}{|l|}{ Partial-error trial } \\
\hline RT & 478 & 35 & 456 & 41 & 528 & 68 & 504 & 49 & 492 \\
\hline EMG latency & 252 & 32 & 233 & 36 & 283 & 61 & 262 & 45 & 257 \\
\hline Erroneous trials $(\%)$ & 5.4 & & 12.8 & & 8.0 & & 17.1 & & 10.8 \\
\hline Partial-error trials $(\%)$ & 11.6 & & 12.0 & & 9.2 & & 9.5 & & 10.6 \\
\hline
\end{tabular}

The latency of partial errors was shorter on compatible than on incompatible trials $[F(1,11)=6.3, p<.03]$ and was shorter in speed- than in accuracy-emphasized conditions $[F(1,11)=14.1, p<.01]$.

No other effect or interaction was significant (all $F \mathrm{~s}<1)$.

\section{Error Rates}

See Table 1, bottom rows. The percentages reported there were arcsine transformed (Winer, 1971) before being submitted to the ANOVA.

Incompatible mapping elicited more errors $[F(1,11)=$ 9.7, $p<.01]$, but fewer partial errors $[F(1,11)=5.7, p<$ $.04]$, than did compatible mapping. Speed-emphasized conditions elicited more errors than did accuracy-emphasized conditions $[F(1,11)=79.6, p<.0001]$, but this effect was not significant for the partial-error rate $(F<1)$. No other interaction was significant (all $F \mathrm{~s}<1$ ).

\section{Sequential Effects ${ }^{2}$}

RTs of pure-correct responses as a function of the subsequent trial. RTs differed depending on the nature of the subsequent trial $[F(2,22)=36.9, p<.001]$. Contrast comparisons indicated that before an erroneous trial, mean RTs were shorter than before a pure-correct trial $[M=376$ vs. $400 \mathrm{msec} ; F(1,11)=49.8, p<.001]$. Before a partial-error trial, mean RTs were shorter than before a pure-correct trial $[M=386$ vs. $400 \mathrm{msec} ; F(1,11)=39.7$, $p \mathrm{~s}<.001]$. In other words, before committing an error or a partial error, subjects speeded up (Figure 2A).

None of the remaining interactions - between the nature of the subsequent trial and compatibility, the nature of the subsequent trial and speed-accuracy instruction, and the nature of the subsequent trial, compatibility, and speedaccuracy instruction - were significant (all $p \mathrm{~s}>.15$ ).

RTs of pure-correct responses as a function of the preceding trial. RTs also differed according to the nature of the preceding trial $[F(2,22)=59.4, p<.001]$. Contrast comparisons indicated that after an erroneous trial, mean RTs were longer than after a pure-correct trial $[M=458$ vs. $386 \mathrm{msec} ; F(1,11)=74.6, p<.001]$. After a partialerror trial, mean RTs were longer than after a pure-correct trial $[M=394$ vs. $386 \mathrm{msec} ; F(1,11)=16, p<.001]$. In other words, after committing an error or a partial error, subjects slowed down (Figure 2B).
The remaining interactions - between the nature of the preceding trial and compatibility, the nature of the preceding trial and speed-accuracy instruction, and the nature of the preceding trial, compatibility, and speed-accuracy instruction-were not significant (all $p \mathrm{~s}>.2$ ).

In addition to this conventional analysis, distribution analyses were performed for the pure-correct responses preceded by either a partial error or a pure-correct response. This was done to check whether the post-partialerror slowing was due to a particular class of trials (the
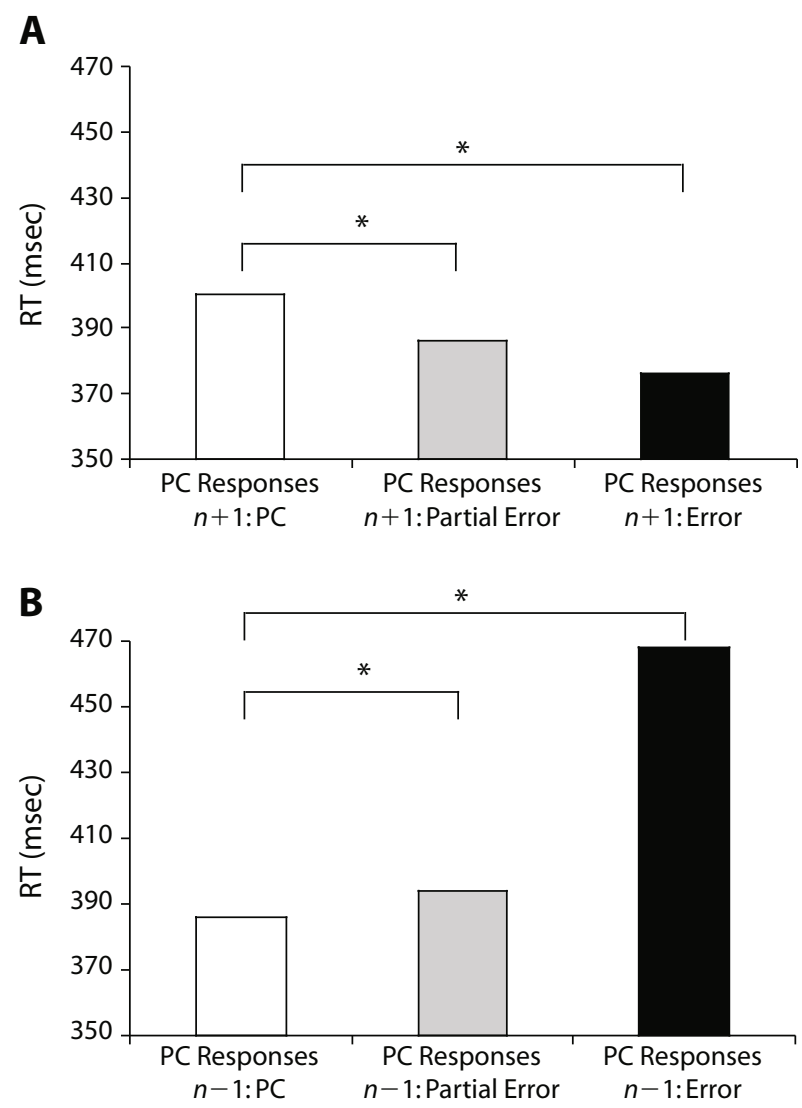

Figure 2. (A) Reaction times (RTs, in milliseconds) of purecorrect (PC) responses as a function of the nature of trial $n+1$. (B) RTs (in milliseconds) of PC responses as a function of the nature of trial $n-1$. * $p<.001$. 
longer). One could imagine that on a small proportion of partial-error trials, the subject thought that he or she had actually pressed the wrong button (i.e., the subject thought that he or she had committed a full error). If this led to a post-error slowing of about 70-80 msec on the next trial, this could explain an overall small post-partial-error slowing averaged across all partial-error trials. ${ }^{3}$ To test this theory, we used the Vincent averaging technique (Ratcliff, 1979; Vincent, 1912). Basically, we binned the RT distribution into five classes of equal size (same number of trials), and the mean of each class was computed. This was done separately for each participant, and the mean values of each class were then averaged across participants. If the post-partial-error slowing was due to a particular class of trials (producing a slowing of 70-80 msec), this slowing should be restricted to the last class, as illustrated in Figure 3A. Figure 3B presents the cumulative density functions for RTs of pure-correct responses preceded by either a pure-correct response or a partial error for each of the experimental conditions.

The RT distributions of pure-correct responses preceded by a pure-correct response and by a partial error are regularly ordered, except for the last class: Post-partial-error slowing is present on the first four classes. To confirm these findings, an ANOVA was performed with nature of the preceding trial (pure-correct response preceded by a pure-correct response or a partial error), speed-accuracy instruction (accuracy, speed), compatibility (compatible, incompatible), and quantile (five classes) as withinsubjects variables. This analysis revealed an effect of the nature of the preceding trial $[F(1,11)=12.82, p<.01]$, indicating that the RTs of pure-correct responses preceded by a partial-error trial were longer than those of purecorrect responses preceded by a pure-correct response. The interaction between the nature of the preceding trial and the quantile was also significant $[F(4,44)=17.95$, $p<.01]$, indicating that the effect of the nature of the preceding trial was significant for each class except the last one. This is completely at variance with the prediction (depicted in Figure 3A) that post-partial-error slowing is an artifact of averaging due to a particular class of trials.

Another hypothesis ${ }^{4}$ is that other sequential effects could explain the post-partial-error slowing: The slowing might be a by-product resulting from alternation or repetition of a correct response from one trial to the next. For this reason, we conducted another analysis on the RTs of purecorrect responses with the following within-subjects variables: speed-accuracy instruction, compatibility (compatible or incompatible), nature of the sequence (alternation or repetition), and nature of the preceding trial (pure correct or partial error). This revealed the classical compatibility effect $[F(1,11)=7.38, p<.05]$, as well as a speedaccuracy instruction effect $[F(1,11)=14.03, p<.01]$. An effect of the nature of the preceding trial $[F(1,11)=5.85$, $p<.05$ ] corresponded to the post-partial-error slowing, and an effect of the nature of the sequence $[F(1,11)=$ $161.74, p<.01]$ indicated that RTs were longer for an alternation of the correct response $(425 \mathrm{msec})$ than for a repetition $(364 \mathrm{msec})$. The interaction between the nature of the sequence and the speed-accuracy instruction was significant $[F(1,11)=9.91, p<.01]$, indicating that the effect of speed-accuracy instruction was much stronger for a repeated $(35 \mathrm{msec})$ than for an alternated $(16 \mathrm{msec})$ correct response. In addition, the interaction between the nature of the sequence and the nature of the preceding trial was significant $[F(1,11)=19.41, p<.01]$, indicating that post-partial-error slowing was observed only for a repetition of the correct response [12 vs. $-2 \mathrm{msec} ; F(1,11)=$ $15.91, p<.01]$.

\section{DISCUSSION}

As Smith and Brewer (1995) showed, before committing an error, subjects speed up: The mean RT on purecorrect trials is shorter before an erroneous trial than before a pure-correct trial. This may be explained as follows. Smith and Brewer showed that on trials preceding an error, the mean RT progressively decreases (below the overall mean RT) until an error is committed: In other words, subjects are in a fast phase. Conversely, on the trial following an error, the mean RT increases and, from the next trial, progressively decreases until it reaches the overall mean RT: In other words, subjects are in a slow phase. Therefore, strategic fluctuations ("micro trade-offs"; Smith \& Brewer, 1995) exist in the speed-accuracy trade-off. From trial to trial, subjects may strategically adopt different positions on the speed-accuracy trade-off function: The smaller the time allowed for processing, the larger the chance of committing an error. We have shown here that before a partial-error trial, subjects also speed up, but the degree of speeding is smaller than before an actual error. This suggests that when subjects are in a very fast phase, the chances of catching a potential error in time are low, and incorrect activation mostly results in a full error. Conversely, when subjects are in a moderately fast phase on the preceding trial (as is the case before a partial error), the chances of catching an error on the current trial are higher, and incorrect activation mostly results in a partial error.

This effect might be explained as follows. Two independent factors may contribute to the likelihood of committing an error: the speed-accuracy strategy, as already proposed by Smith and Brewer (1995), and the level of online response monitoring. The higher the level of response monitoring on a given trial, the greater the chance of catching an error before it is committed.

It has been proposed that a function of the executive control system on a given trial is to trigger strategic adjustments on the subsequent trial (Botvinick et al., 2001). Thus, it is possible that a decrease of the efficiency of the executive control system sometimes leads to a decrease of response monitoring on the following trial. Such a decrease in executive control may augment the likelihood of committing an error on the following trial, by decreasing the possibility for the response-monitoring system to detect and/or correct an incorrect activation. Indeed, Ridderinkhof, Nieuwenhuis, and Bashore (2003) have suggested that the efficiency of the executive control system is lower before an error (see also Allain, Carbonnell, Falkenstein, Burle, \& Vidal, 2004). We propose that the 


\section{A Hypothetical Distribution}

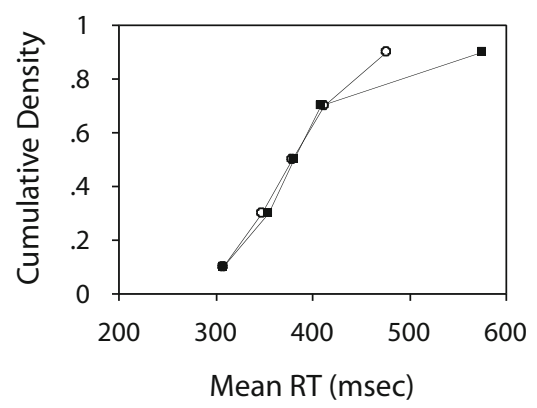

B

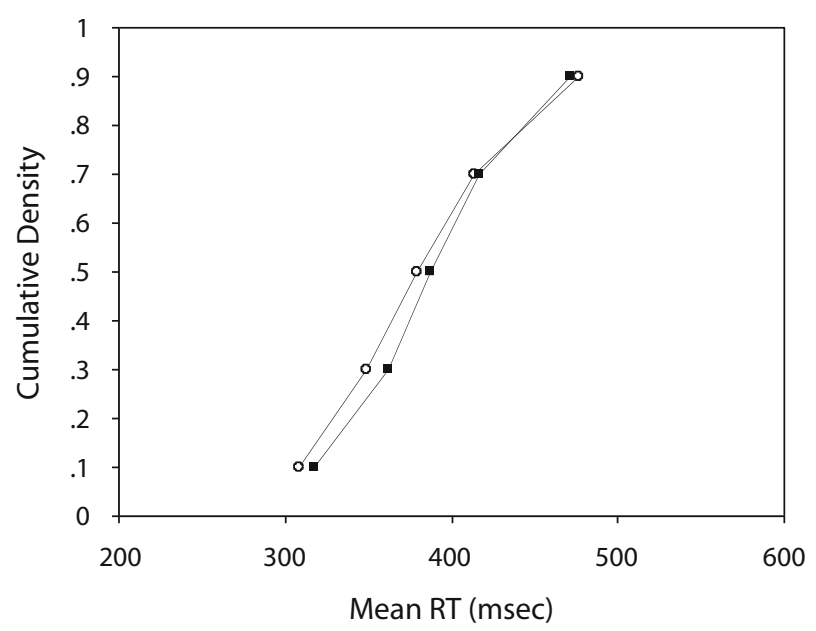

Speeded-Compatible

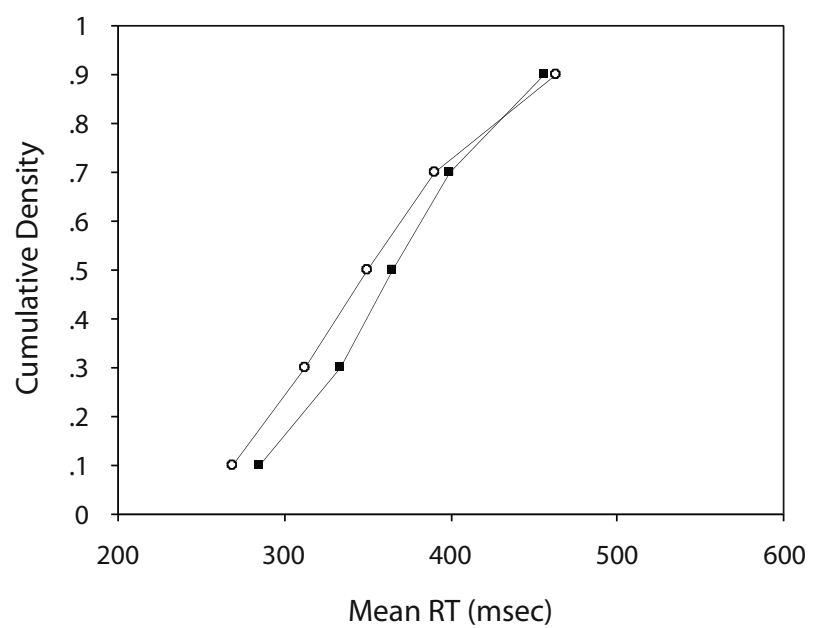

Accurate-Incompatible

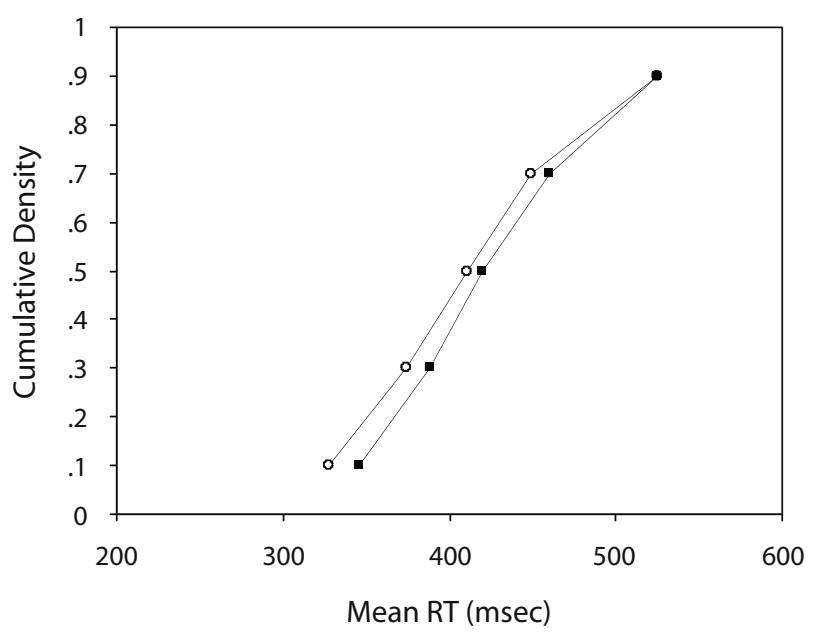

Speeded-Incompatible

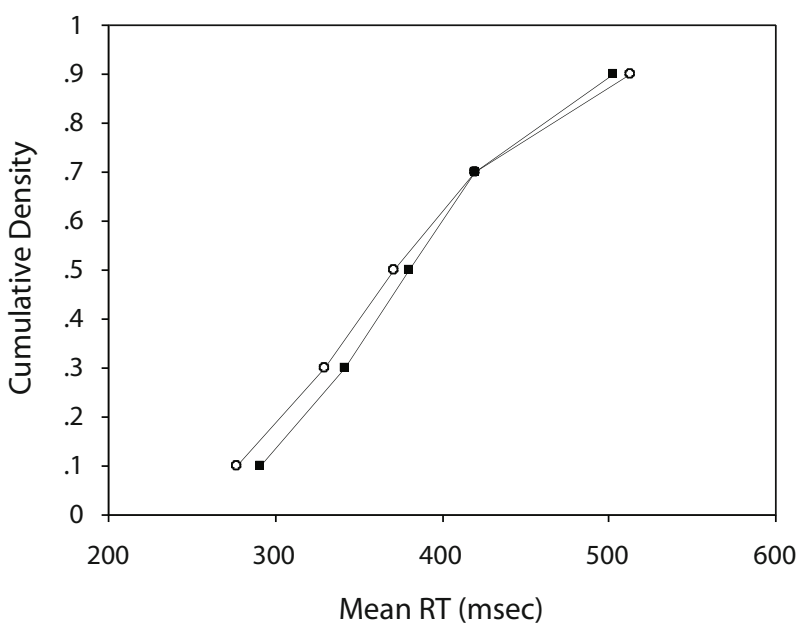

Figure 3. (A) Hypothetical cumulative density functions of reaction times (RTs) for pure-correct responses preceded by a partialerror trial (post-partial-error) and by a pure-correct response (post-pure-correct). (B) Cumulative density functions of RTs for purecorrect responses preceded by a partial error and by a pure-correct response, for all experimental conditions.

more efficient the performance (the shorter RTs become while still maintaining a correct response), the lower the level of executive control in the given trial, the lower the monitoring of the response on the next trial, and the lower the chances of catching an error on this next trial. There- fore, if subjects are very fast before a given trial (pre-error speeding), the likelihood of detecting and correcting incorrect activation in progress on the current trial is low, and therefore the probability of committing an error is high. If a subject is moderately fast before a given trial 
(pre-partial-error speeding), the likelihood of detecting and correcting ongoing incorrect activation on the current trial is higher, and the probability of producing a partial error is high.

As shown by Rabbitt (1966), subjects slow down after committing an error: The mean RT on pure-correct trials is longer after an erroneous trial than after a pure-correct trial. We have shown that after a partial-error trial, subjects also slow down. This effect is small $(8 \mathrm{msec})$ but reliable and stable across subjects and conditions, since it is not affected by compatibility or the speed-accuracy instruction. It is well established that RTs are autocorrelated (Laming, 1979a); that is, if the RT happens to be long (or short) on trial $n$, the RT of trial $n+1$ will also be prone to be long (or short). RTs of correct trials containing a partial error are longer than those of pure-correct trials. Therefore, one could imagine that the short RT increase observed after partial errors is just a by-product of RT autocorrelation. According to this view, there is no "true" post-partial-error slowing. However, this interpretation is not tenable, because post-partial-error slowing occurs only when there is a repetition of the correct response; according to the autocorrelation explanation, post-partialerror slowing should also be observed after a response alternation, which is not the case in the present data set.

The size of this effect is not of the same order of magnitude as the post-error slowing $(72 \mathrm{msec})$. A possible explanation can be found in the data of Burns (1971), who showed that post-error slowing decreases as the interstimulus interval increases. In the present study, the responsestimulus interval was constant, including on partial-error trials. However, if we consider the partial error itself (and not the correct response on that trial), the delay separating the partial error from the next response signal was longer $(150 \mathrm{msec})$ than the response-stimulus interval for overt errors. Therefore, the small size of post-partial-error slowing in comparison with post-error slowing may be due, at least in part, to an increase in the response-stimulus interval for partial errors.

It should also be noted that on a partial-error trial, subjects provide the correct response before the subsequent trial; on overt error trials, however, since subjects are encouraged not to correct their errors, there is no such intermediate response. The presence of an intermediate correct response on partial-error trials could also explain why post-partial-error slowing is much smaller than posterror slowing. Conceptually, this explanation makes sense only if we consider partial errors as errors that are detected, inhibited, and corrected in time: During a trial, an executive control system monitors the execution of the response. If the incorrect response is activated, the control system detects it, and an active process attempts to inhibit this incorrect activation. If this process succeeds, the incorrect activation is actively stopped, and a partial error occurs, followed by the correct response; in this case, partial errors correspond to small errors corrected in time, as proposed by Burle, Possamaï, Vidal, Bonnet, and Hasbroucq (2002). Furthermore, it is important to note that in this case, the response-monitoring system is very sensitive, since on partial errors there is neither a click nor somatosensory information indicating a response; in other words, there is no available external feedback.

When this process fails to inhibit the incorrect activation, an overt erroneous response is emitted; a trace of the inhibitory process still persists at the peripheral level as a reduction of the EMG burst (Allain, Carbonnell, Burle, Hasbroucq, \& Vidal, 2004). Therefore, whether the outcome is an overt or partial error, executive control adjustments will occur (Burle et al., 2002; Laming, 1979b; Rabbitt, 1966).

Another point deserves some comment. It could be argued that on correct trials containing a partial error, inhibition processes that are needed to stop the erroneous response on trial $n$ spill over into trial $n+1$, thus explaining the RT increase. According to this explanation, posterror slowing does not reflect "true" adjustments but is a result of inhibitory processes occurring on the preceding trial. However, post-partial-error slowing occurs only for a repetition of the correct response - that is, for an alternation between the partial error on trial $n$ and the correct response on trial $n+1$. This result is exactly opposite to the predictions of the spill-over hypothesis. Hence, postpartial-error slowing does seem to correspond to true selective adjustment. In other words, the supervisory system triggers post-partial-error adjustments only when, in the following trial, the stimulus-response association is identical to the previous, nearly missed, one. Therefore, the supervisory system seems able to act in a very selective manner.

Whereas pre-error speeding and pre-partial-error speeding are qualitatively (but not quantitatively) equivalent, the selectivity of post-partial-error slowing indicates that post-error and post-partial-error slowing are qualitatively (and quantitatively) different. This does not necessarily mean that their mechanisms are different, but further experiments will be necessary to elucidate the functional significance of this selectivity.

Finally, it should be noted that few studies take partial errors into account, even though such data probably reveal cognitive processes to which one has no access unless EMG recordings are performed. Partial errors should not be neglected, because they may help constrain the available models of action monitoring. For example, the results of the present study seem to be compatible with the conflict loop theory (Botvinick et al., 2001): In a partial-error trial, the level of conflict is high (but lower than on an error trial) because two responses are coactivated in the same trial (Yeung, Botvinick, \& Cohen, 2004). According to this model, the conflict triggers adjustments, such as slowing, on the subsequent trial. However, we show that the post-partial-error slowing occurs only for a repetition of the correct response. If we consider that conflict is supposed to occur on all partial-error trials, independent of the nature of the subsequent response (alternation or repetition), the present form of the conflict model does not account for our data.

Another example, still in the context of the conflict loop theory, can be found in the work of Carter et al. (1998). These authors showed that the anterior cingulate cortex, where the conflict-monitoring module would be located, is 
activated when an error is committed and for certain correct responses. But they did not distinguish pure-correct responses from correct responses preceded by a partial error. If partial errors are small (corrected) errors, Carter et al.'s interpretation that conflict is present on correct trials is not warranted. Alternatively, one could interpret their cingulate activation on correct responses as due to the presence of partial errors. To fully prove the presence of conflict for correct responses, this should be established with partial-error-free response data. We conclude that considering partial errors as small corrected errors may help constrain current models of executive control. Authors have previously argued that partial errors are small corrected errors (Allain, Carbonnell, Burle, et al., 2004; Burle, Allain, Vidal, \& Hasbroucq, 2005); here, we provide additional empirical support for this view, showing that sequential effects are similar for partial and for full errors.

\section{AUTHOR NOTE}

Correspondence related to this article may be sent to S. Allain, Institut de Médecine Navale du Service de Santé des Armées, BP 610083800 , Toulon Armée (e-mail: allainsonia@hotmail.com).

\section{REFERENCES}

Allain, S., Carbonnell, L., Burle, B., Hasbrouce, T., \& Vidal, F. (2004). On-line executive control: An electromyographic study. Psychophysiology, 41, 113-116.

Allain, S., Carbonnell, L., Falkenstein, M., Burle, B., \& Vidal, F. (2004). The modulation of the Ne-like wave on correct responses foreshadows errors. Neuroscience Letters, 372, 161-166.

Botvinick, M. M., Braver, T. S., Barch, D. M., Carter, C. S., \& Cohen, J. D. (2001). Conflict monitoring and cognitive control. Psychological Review, 108, 624-652.

Burle, B., Allain, S., Vidal, F., \& HasbroucQ, T. (2005). Sequential compatibility effects and cognitive control: Does conflict really matter? Journal of Experimental Psychology: Human Perception \& Performance, 31, 831-837.

Burle, B., Possamaï, C.-A., Vidal, F., Bonnet, M., \& Hasbrouce, T. (2002). Executive control in the Simon effect: An electromyographic and distributional analysis. Psychological Research, 66, 324-336.

BURNS, J. T. (1971). Error-induced inhibition in a serial reaction time task. Journal of Experimental Psychology, 90, 141-148.

Carter, C. S., Braver, T. S., Barch, D. M., Botvinick, M. M., Noll, D., \& Cohen, J. D. (1998). Anterior cingulate cortex, error detection, and the online monitoring of performance. Science, 280, 747-749.

Coles, M. G. H., Gratton, G., Bashore, T. R., Eriksen, C. W., \& Donchin, E. (1985). A psychophysiological investigation of the continuous flow model of human information processing. Journal of Experimental Psychology: Human Perception \& Performance, 11, 529-553.

Eriksen, C. W., Coles, M. G. H., Morris, L. R., \& O'Hara, W. P. (1985). An electromyographic examination of response competition. Bulletin of the Psychonomic Society, 23, 165-168.

HasbroucQ, T., Possamaï, C.-A., Bonnet, M., \& Vidal, F. (1999). Effect of the irrelevant location of the response signal on choice reaction time: An electromyographic study in humans. Psychophysiology, 36, 522-526.

LAMING, D. (1979a). Autocorrelation of choice-reaction times. Acta Psychologica, 43, 381-412.

LAMING, D. (1979b). Choice reaction performance following an error. Acta Psychologica, 43, 199-224.

RabBitT, P. M. A. (1966). Errors and error correction in choice-response tasks. Journal of Experimental Psychology, 71, 264-272.

RATCLIFF, R. (1979). Group reaction time distributions and an analysis of distribution statistics. Psychological Bulletin, 86, 446-461.

RidderinkHof, K. R., Nieuwenhuis, S., \& Bashore, T. R. (2003). Errors are foreshadowed in brain potentials associated with action monitoring in cingulate cortex in humans. Neuroscience Letters, 348, 1-4.

Smid, H. G. O. M., Mulder, G., \& Mulder, L. J. M. (1990). Selective response activation can begin before stimulus recognition is complete: A psychophysiological and error analysis of continuous flow. Acta Psychologica, 74, 169-201.

Smith, G. A., \& Brewer, N. (1995). Slowness and age: Speed-accuracy mechanisms. Psychology \& Aging, 10, 238-247.

Staude, G. H. (2001). Precise onset detection of human motor responses using a whitening filter and the log-likelihood-ratio test. IEEE Transactions on Biomedical Engineering, 48, 1292-1305.

Van Boxtel, G. J. M., Geraats, L. H. D., Van den Berg-Lenssen, M. M. C., \& Brunia, C. H. M. (1993). Detection of EMG onset in ERP research. Psychophysiology, 30, 405-412.

VINCENT, S. B. (1912). The function of vibrossae in the behavior of the white rat. Behavioral Monographs, 1, 1-181.

WINER, B. J. (1971). Statistical principles in experimental design (2nd ed.). New York: McGraw-Hill.

Yeung, N., Botvinick, M. M., \& Cohen, J. D. (2004). The neural basis of error detection: Conflict monitoring and the error-related negativity. Psychological Review, 111, 931-959.

\section{NOTES}

1. Even though subjects were told that correcting a response was not helpful, they still did so for $20 \%$ of the errors.

2 . We did not perform an analysis on the percentage of errors following or preceding an error, since the percentage of these trials was too low to allow for reliable analysis.

3. This interesting point has been raised by the editor.

4. We thank an anonymous reviewer for raising this question.

(Manuscript received December 18, 2007; revision accepted for publication November 26, 2008.) 